\title{
Platelet-rich plasma exhibits beneficial effects for rheumatoid arthritis mice by suppressing inflammatory factors
}

\author{
SHICHAO TONG ${ }^{1}$, CHANGQING ZHANG ${ }^{1}$ and $\mathrm{JI} \mathrm{LIU}^{2}$ \\ ${ }^{1}$ Department of Orthopedics, Shanghai Sixth People's Hospital; ${ }^{2}$ Department of Orthopedics, Shanghai \\ Ruijin Hospital, School of Medicine, Shanghai Jiaotong University, Shanghai 200233, P.R. China
}

Received April 19, 2016; Accepted March 21, 2017

DOI: $10.3892 / \mathrm{mmr} .2017 .7091$

\begin{abstract}
Platelet-rich plasma (PRP) is a multifunctional blood product containing highly concentrated platelets, and various cell growth factors which promote cell proliferation and differentiation. PRP exhibited benefits in injurious articular cartilage repair and the removal of inflammatory factors in clinical studies. Rheumatoid arthritis (RA) is an autoimmune disease manifesting primarily as inflammatory arthritis, which is associated with notable morbidity in humans. In the present study, the therapeutic effects and primary mechanism of PRP on a type II collagen-induced arthritis (CIA) mouse model was investigated. Inflammatory factors interleukin (IL)-6, IL-8, IL-17, IL-1 $\beta$, tumor necrosis factor (TNF)- $\alpha$ and interferon (IFN)- $\gamma$ were analyzed in PRP and PBS-treated groups. Vascular endothelial growth factor (VEGF), platelet-derived growth factor (PDGF), insulin-like growth factor (IGF)-1 and transforming growth factor (TGF) $-\beta$ expression in peripheral whole blood was additionally analyzed. The therapeutic efficacy of PRP for RA mice was evaluated using clinical arthritis scores. The results of the present study demonstrated that treatment with PRP alleviated arthritis, and reduced humoral and cellular immune responses, leading to beneficial effects on histological parameters as observed using joint tissue histological staining. CIA mice treated with PRP exhibited downregulated expression of IL-6, IL-8, IL-17A, IL-1 $\beta$, TNF- $\alpha$, receptor activator for nuclear factor- $\mathrm{kB}$ and IFN- $\gamma$ in inflammatory tissue. In addition, VEGF, PDGF, IGF-1 and TGF- $\beta$ expression in peripheral whole blood was increased following treatment with PRP. The serum concentration of anti-collagen antibody was decreased in PRP-treated CIA mice. In conclusion, CIA mice treated with PRP exhibited beneficial effects, including decreased joint inflammation, cartilage destruction and bone damage, and increased repair
\end{abstract}

Correspondence to: Professor Changqing Zhang, Department of Orthopedics, Shanghai Sixth People's Hospital, Shanghai Jiaotong University, 600 Yishan Road, Shanghai 200233, P.R. China E-mail: angcq@sjtu.edu.cn; zhangchangqingsci@163.com

Key words: platelet-rich plasma, rheumatoid arthritis, type II collagen-induced arthritis mouse model of joint tissue. The results of the present study suggested that PRP may be an effective therapeutic agent for RA.

\section{Introduction}

Platelet-rich plasma (PRP) is a prominent biomedical agent that has demonstrated efficacy in the treatment of femoral head damage, cartilage disorders and rheumatoid arthritis (1). PRP is a blood product rich in cytokines, growth factors and other bio-active molecules, which is obtained from autologous peripheral blood mononuclear cells (PBMCs) and has been used in innovative treatment protocols (2). The use of PRP may not lead to the side effects and treatment-associated adverse events of classical drug products (3). Previous studies demonstrated that an intravenous injection of PRP was able to repair tendons and damaged articular bone, and contribute to inflammatory elimination, which may serve an important role in the morphology, collagen microarchitecture and subsequent mechanical properties of the injected vein $(2,4,5)$. As demonstrated in previous studies, treatment with PRP results in marked clinical improvements in patients with symptomatic osteoarthritis of the knee (6-8). Rheumatoid arthritis (RA) is an intractable and chronic inflammatory autoimmune disease characterized by the release of inflammatory cytokines, leading to infiltration of autoimmune cells into the synovium and subsequent joint damage (9).

Innate and adaptive immune responses of autoantibodies have been demonstrated to serve a role in the dysregulated expression of inflammatory cytokines, which may target the synovium and subsequently result in joint disease $(9,10)$. RA is an autoimmune disease characterized by chronic inflammation of the joints (11). RA is associated with inflammation of synovial joints, primarily the hands and feet, in addition to systemic extra-articular inflammation (12). Inflammatory cytokines are identified as a complex regulatory signaling network in RA, which is mediated by various intracellular kinase signaling pathways to regulate the recruitment, stimulation, activation and function of autoimmune cells (13). Although the causes of RA are not completely understood, laboratory and clinical evidence suggests that pro-inflammatory cytokines, particularly tumor necrosis factor (TNF), serve an important role in its pathogenesis $(14,15)$. It has been hypothesized that inhibiting the TNF pathway using a specific anti-TNF- $\alpha$ antibody may interrupt the inflammatory process to break the cycle of 
inflammation and limit joint damage $(16,17)$. A previous study has indicated that intracellular signaling pathways mediated by inflammatory cytokines may underlie the mechanism of initiation, development and aggravation of RA, and may represent a key interaction in the cytokine-induced RA network (18). Therefore, therapeutic agents targeted to inflammatory cytokines may be beneficial for RA treatment by modulating and neutralizing cytokines, leading to inhibition of inflammatory responses.

In the present study, the efficacy and mechanism of action of PRP was investigated in a type II collagen-induced arthritis (CIA) mouse model. Inflammatory factors were analyzed in PRP and PBS-treated groups. The therapeutic efficacy of PRP for RA mice was evaluated using clinical arthritis scores. The effects of PRP on the tyrosine protein kinase JAK (JAK) signaling pathway, and on immune and inflammatory responses in RA mice, were analyzed.

\section{Materials and methods}

Ethics statement. The present study was performed in accordance with the recommendations in the Guide for the Care and Use of Laboratory Animals of the National Institutes of Health (Bethesda, MA, USA) (19). The protocol was approved by the Chinese Association for Laboratory Animal Sciences (Beijing, China), Animal Health Products (Beijing, China), Committee on the Ethics of Animal Experiments Defense Research (Beijing, China) and Development China (Beijing, China), and Animal Experiments of Shanghai Sixth People's Hospital (approval no. SCXK-2014-1243). All surgery and euthanasia was performed under sodium pentobarbital anesthesia, and all efforts were made to minimize suffering.

Cells and reagents. Hela cells were purchased from the American Type Culture Collection (Manassas, VA, USA). Mouse RA synoviocytes were donated by Harbin Medical University (Harbin, China). Culture flasks, medium, chemicals and reagents were purchased from recognized commercial suppliers.

Detection of cytokine gene expression. Mouse RA synoviocytes were cultured in Dulbecco's modified Eagle's medium (DMEM; Sigma-Aldrich; Merck KGaA, Darmstadt, Germany) with $10 \%$ fetal bovine serum (FBS) in 6-well plates. At $90 \%$ confluence, cells were stimulated with $50 \mu \mathrm{l} / \mathrm{ml}$ PRP (Sigma-Aldrich; Merck KGaA), or $50 \mu \mathrm{l} / \mathrm{ml}$, dexamethasone (DEX; Sigma-Aldrich; Merck KGaA) or PBS for $12 \mathrm{~h}$ at $37^{\circ} \mathrm{C}$ in a $5 \% \mathrm{CO}_{2}$ humidified atmosphere. RA synoviocytes without antigen and antibody were used as negative controls. Hela cells incubated with DEX, PRP or PBS for $12 \mathrm{~h}$ were used as positive controls. The cells were collected following $12 \mathrm{~h}$ of incubation, and total RNA was extracted to detect the expression of mRNA for IL- 6 and IL- 8 by using the reverse transcription-quantitative polymerase chain reaction (RT-qPCR).

Antibody-dependent cell-mediated cytotoxicity (ADCC) and complement-dependent cytotoxicity (CDC) assays. PMBCs were prepared by Ficoll-Hypaque density gradient (Sigma-Aldrich; Merck KGaA) centrifugation (2,000 x g at $4^{\circ} \mathrm{C}$ for $\left.10 \mathrm{~min}\right)$. ADCC and CDC activities of PRP were measured by lactate dehydrogenase (LDH) assay (Merck KGaA, Darmstadt, Germany), which measures the activity of $\mathrm{LDH}$ released from the cytosol of damaged cells. Hela cells stably expressing transmembrane TNF- $\alpha$ were incubated with different concentrations $(0.2-35 \mu \mathrm{g} / \mathrm{ml})$ of anti-human TNF antibody for $1 \mathrm{~h}$ in assay medium (DMEM $+5 \%$ FBS) in a $5 \% \mathrm{CO}_{2}$ incubator at $37^{\circ} \mathrm{C}$, followed by the addition of human PBMCs (Shanghai Sixth People's Hospital, Shanghai, China) as effector cells (ADCC assay, effector to target ratio $=20: 1$ ) or human complement-rich serum (Quidel Corporation, San Diego, CA, USA) (CDC assay, 1.25\% v/v). Following an additional incubation at $37^{\circ} \mathrm{C}$ for $16 \mathrm{~h}$ for the ADCC assay and $5 \mathrm{~h}$ for the CDC assay, $100 \mu \mathrm{l}$ supernatant from each well was transferred into a flat-bottomed 96-well plate. LDH substrate $(100 \mu l)$ was added to each well and incubated for $30 \mathrm{~min}$ at room temperature in the dark. The absorbance of the samples was measure at $490 \mathrm{~nm}$ with a microplate reader.

$R N A$ isolation and $R T-q P C R$. Total RNA was extracted from RA synoviocytes or synovial tissue using RNAzol (Sigma-Aldrich; Merck KGaA), and RNase-free DNase (Sigma-Aldrich; Merck KGaA) was used to digest total RNA at $37^{\circ} \mathrm{C}$ for $15 \mathrm{~min}$. An RNeasy kit (Sigma-Aldrich; Merck KGaA) was used to purify RNA and adjust its concentration to $1 \mu \mathrm{g} / \mu \mathrm{l}$. A total of $1 \mu \mathrm{g}$ RNA was reverse-transcribed using the Thermo Script RT-qPCR system (Invitrogen; Thermo Fisher Scientific, Inc., Waltham, MA, USA). The concentration of RNA was quantified using spectrophotometry at $260 \mathrm{~nm}$ (Smart Spec TM 3000; Bio-Rad Laboratories, Inc., Hercules, CA, USA). Total RNA in the RA synoviocytes and synovial tissue of CIA mice was denatured by incubating for $5 \mathrm{~min}$ at $70^{\circ} \mathrm{C}$ with $4 \mu \mathrm{M}$ oligo(dT) primer and reverse-transcribed using a final concentration of $0.5 \mathrm{mM}$ dNTP, $40 \mathrm{U} / \mu 1 \mathrm{RNaseOUT}$ (Sigma-Aldrich; Merck KGaA), $0.01 \mathrm{M}$ dithiothreitol, and $10 \mathrm{U} / \mu 1$ Thermo Scriptreverse transcriptase (mRNA expression in RA synoviocytes or synovial tissue). Reverse transcription was performed by incubating at $42^{\circ} \mathrm{C}$ for $180 \mathrm{~min}$. The obtained cDNA was diluted 10 -fold with distilled $\mathrm{H}_{2} \mathrm{O}$ and $10 \mu \mathrm{l}$ was used for amplification. Specific primer sets for interleukin (IL)-6, IL-8, matrix metalloproteinase (MMP)-3, IL-17A, IL-1 $\beta$, TNF- $\alpha$, receptor activator for nuclear factor- $\kappa \mathrm{B}$ ligand (RANKL), VEGF, PDGF, IGF-1, TGF- $\beta$ and interferon- $\gamma($ IFN- $\gamma)$ were generated. All PCR primers are listed in Table I. The PCR was performed using the Light Cycler Fast Start DNA SYBR Green I kit (Roche Diagnostics, Basel, Switzerland) according to the protocol provided with the parameter-specific kits (45 amplification cycles, denaturation at $96^{\circ} \mathrm{C}$, primer annealing at $68^{\circ} \mathrm{C}$ with touchdown to $58^{\circ} \mathrm{C}$, and applicant extension at $\left.72^{\circ} \mathrm{C}\right)(20)$.

Animal study. DBA/1J male mice at 8-10 weeks of age (30-35 g) were purchased from Shanghai SLAC Laboratory Animals Co., Ltd. (Shanghai, China). Mice were maintained in a climate-controlled $20-25^{\circ} \mathrm{C}$ environment with a $12-\mathrm{h}$ light/dark cycle. The induction of type II CIA was achieved as previously described (21), by the subcutaneous injection of $2 \mathrm{mg}$ collagen (ModiQuest Research, Oss, Netherlands) in each mouse ( $n=9 /$ group) (22). Clinical arthritis scores 
Table I. Sequences of primers used.

Sequence

\begin{tabular}{lll}
\cline { 2 - 3 } Gene name & \multicolumn{1}{c}{ Reverse } & \multicolumn{1}{c}{ Forward } \\
\hline TNF- $\alpha$ & 5'-TCCAGACTTCCTTGAGACA-3' & 5'-GGCGATTACAGACACAACT-3' \\
IL-1 $\beta$ & 5'-GGCTGCTTCCAAACCTTTGA-3' & 5'-GAAGACACGGATTCCATGGT-3' \\
IL-6 & 5'-GTGAGGAACAAGCCAGAG-3' & 5'-TGACCAGAAGAAGGAATGC-3' \\
IL-17A & 5'-ATGCACAGCCACCGCGACTT-3' & 5'-CTTCATGACTGCCTCCAAGTAG-3' \\
MMP-3 & 5'-GCCCTGGAACTCACACGACA-3' & 5'-TTGGAAACTCACACGCCAGAAG-3' \\
RANKL & 5'-AAGGCGAGAGATTCTTTCCCT G'-3' & 5'-ACTGGGGACAATTCACTAGAGC-3' \\
VEGF & 5'-TGCATTCACATTGTGCTGCTGTAG-3' & 5'-GCAGATTATGCGGATCAAACC-3' \\
IGF-1 & 5'-ATCCATGTGTGACCATGAGGAAATG-3' & 5'-TCGGCTAGTTAGGGTACACTTC-3' \\
TGF- $\beta$ & 5'-ATCCATGTGTGACCATGAGGAAATG-3' & 5'TCGGCTAGTTAGGGTACACTTC-3' \\
PDGF & 5'-ATGAGAGTGAGATCGAAGGCA-3' & 5'-CGGCAAGGTATGATGGCAGAG-3' \\
IFN- $\gamma$ & 5'-CACCCTCTGTCACCTGCTCAA-3' & 5'-ATGGCGCTGAGAAGACTTGGT-3' \\
$\beta$-actin & 5'-CGGAGTCAACGGATTTGGTC-3' & 5'-AGCCTTCTCCATGGTCGTGA-3' \\
\hline
\end{tabular}

TNF, tumor necrosis factor; IL, interleukin; MMP, matrix metalloproteinase; RANKL, nuclear factor- $\kappa \mathrm{B}$ ligand; VEGF, vascular endothelial growth factor; IGF, insulin-like growth factor; TGF, transforming growth factor; PDGF, platelet-derived growth factor; IFN, interferon.

were evaluated using a scale of $0-2$ for each paw, for a total score of 8 . Paws were assigned a clinical score based on the index (ModiQuest Research scoring method): $0=$ normal; $0.25=1-2$ swollen toes; $0.5=3-4$ swollen toes; $0.75=$ slightly swollen footpad/ankle; $1=$ swollen footpad/ankle; $1.25=1-2$ swollen toes and swollen footpad/ankle; $2.0=$ swollen toes, footpad and ankle. At days 30 and 70 following the first injection, blood samples were taken from each mouse and anti-CII immunoglobulin ( $\mathrm{Ig}) \mathrm{G}$ antibodies in sera were detected using a mouse anti-type II collagen IgG assay kit (CDT31102; Cayman Chemical Company, Ann Arbor, MI, USA) according to the manufacturer's protocol. The treatment was started on day 30 following the initial injection. Mice were administered $10 \mathrm{mg} / \mathrm{kg}$ PRP or PBS by subcutaneous injection every 4 days for a total of 9 doses, and were sacrificed on day 72 (14).

Histopathological analysis. The RA mice were sacrificed on day 72 , and the knee joints (condyle) were obtained and subsequently fixed in $10 \%$ formalin for $2 \mathrm{~h}$ at $37^{\circ} \mathrm{C}$. The joints were decalcified and embedded in paraffin. The ankle joints were stained with hematoxylin and eosin $(1 \%)$ for $10 \mathrm{~h}$ at $37^{\circ} \mathrm{C}$ to analyze the efficacy of PRP for CIA RA mice. The severity of arthritis in the joints was scored on a scale of 0-5 as described in a previous study (23).

Statistical analysis. All date were expressed as mean and standard deviation of triplicate dependent experiments. Statistical significance was determined using the Student's t-test and Microsoft Excel software (2010; Microsoft Corporation, Redmond, WA, USA). One-way analysis of variance (ANOVA), two-way ANOVA, Kaplan-Meier estimators and log rank statistical analyses were performed using SPSS software (version 19.0; IBM SPSS, Armonk, $\mathrm{NY}, \mathrm{USA}$ ). $\mathrm{P}<0.05$ was considered to indicate a statistically significant difference.

\section{Results}

Functional analysis of PRP in synovial cells in vitro. The in vitro function of PRP in regulating inflammatory factors in synovial cells was assessed. Gene expression of IL-6 and IL-8 in Hela cells and human fibroblast-like synovial cells was measured by RT-qPCR. The results in Fig. 1 demonstrated that PRP stimulated Hela and fibroblast-like synovial cells to increase gene expression of IL-6 and IL-8, while IL-1 $\beta$, IL-17A, TNF- $\alpha$ and IFN- $\gamma$ mRNA expression was downregulated compared with the DEX and control groups. The results of the present study indicated that treatment with PRP decreased cytokine production in vitro.

$A D C C$ and $C D C$ mediated by PRP. A previous study demonstrated that inflammatory factors may be able to affect the progression of RA through ADCC or CDC mechanisms, and that this may lead to various clinical effects (24). In the ADCC assay, the results presented in Fig. 2A demonstrated that $34 \%$ of the IL-1R-expressing Hela target cells were lysed by PRP at a concentration of $10 \mu \mathrm{g} / \mathrm{ml}$. In the CDC assay, the result in Fig. 2B demonstrated that PRP was also capable of lysing IL-1R-expressing Hela cells in the presence of human complement. These data indicated that PRP was able to mediate ADCC and CDC effects upon binding to transmembrane IL-1R expressed on the cell surface, and therefore exhibits the potential to be developed into a more effective IL-1R-neutralizing agent in RA therapy. In addition, all synovial specimens in RA mice treated by PRP, DEX or PBS were analyzed. The results demonstrated that hypertrophy and leukocyte infiltration were suppressed following 4 weeks of treatment with PRP, compared with DEX- and PBS-treated groups (Fig. 2C and D).

Therapeutic effects of PRP for CIA RA mice. In order to assess the therapeutic effects of PRP on RA development, the CIA mouse model was used. The experimental mice were 
A

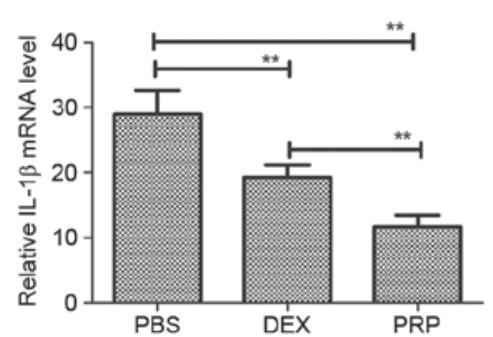

D

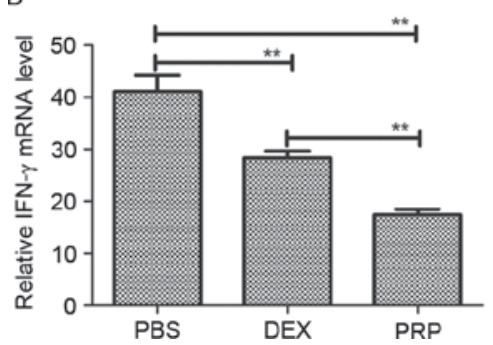

B

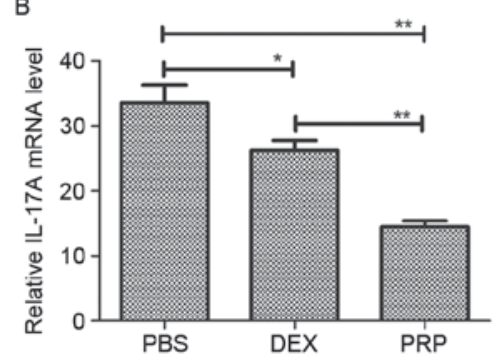

E

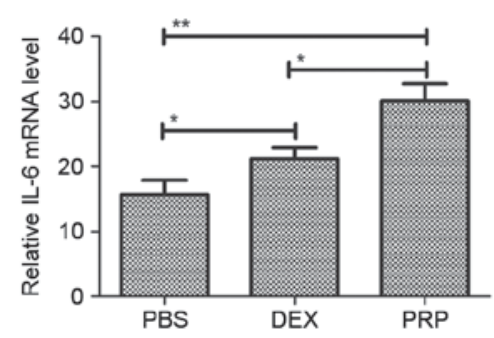

C
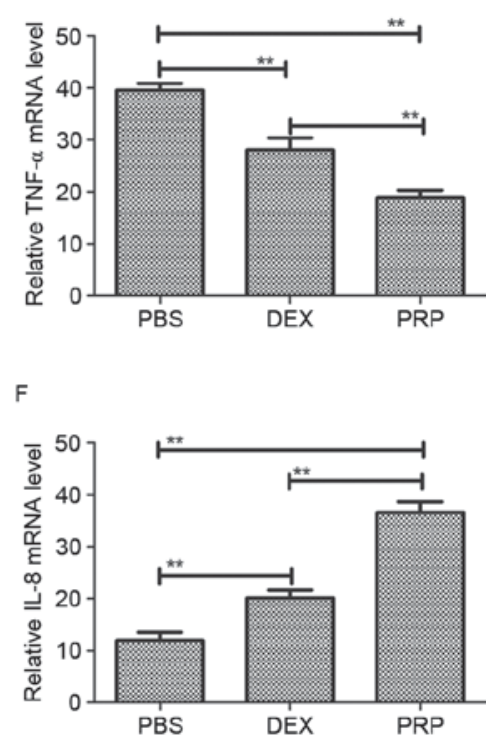

Figure 1. RT-qPCR analysis of the in vitro biological activity of PRP. Gene expression of (A) IL-1 $\beta$, (B) IL-17A, (C) TNF- $\alpha$, (D) IFN- $\gamma$, (E) IL-6 and (F) IL-8 was measured by RT-qPCR. Expression of each gene was calculated relative to the expression of housekeeping gene $\beta$-actin. Data are presented as the mean \pm standard error of the mean of triplicate samples. Student's paired two-tailed t-test; ${ }^{*} \mathrm{P}<0.05,{ }^{* *} \mathrm{P}<0.01$. PRP, platelet-rich plasma; DEX, dexamethasone; IL, interleukin; IFN, interferon; TNF, tumor necrosis factor; RT-qPCR, reverse transcription-quantitative polymerase chain reaction.

A



C

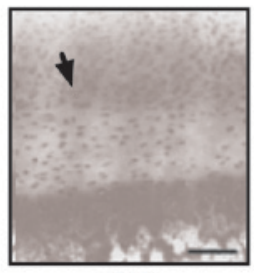

PBS

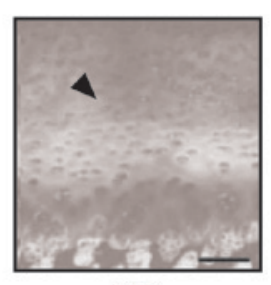

DEX
B

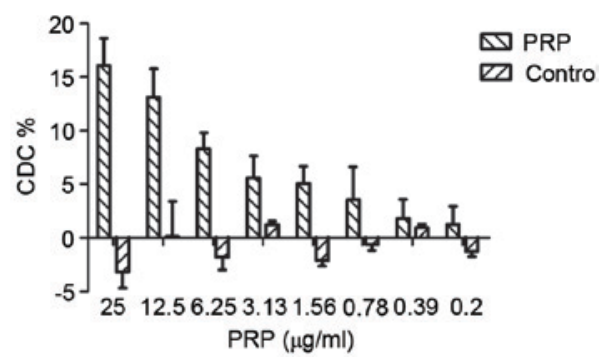

D

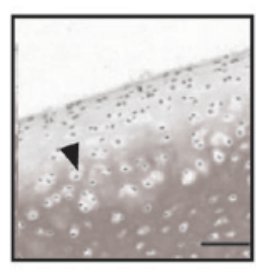

PBS

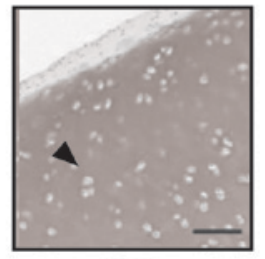

PRP

Figure 2. Efficacy of PRP on ADCC, CDC, proteoglycan and type II collagen content. The efficacy of PRP was analyzed by (A) ADCC and (B) CDC assays. Transmembrane TNF- $\alpha$-expressing Hela cells were incubated in the presence of different concentrations of PRP for 30 min. Subsequently, peripheral blood mononuclear cells for ADCC, or human complement-rich serum for CDC, were used as effector cells and the source of complement, respectively, and transmembrane TNF- $\alpha$-expressing cells were used as targets. (C) Recovery of the loss of cartilage-specific protein (arrowheads) following treatment with PRP demonstrated by staining of cartilage from the joints of rheumatoid arthritis mice (magnification, x200). (D) Alterations in type II collagen at the arthritis induction site (arrowheads) following treatment with PRP in RA mice. ADCC, antigen-dependent cell-mediated cytotoxicity; CDC, complement-derived cytotoxicity; PRP, platelet-rich plasma; TNF, tumor necrosis factor; DEX, dexamethasone.

recorded, and the arthritis score was evaluated every 4 days. As presented in Fig. 3A, treatment with PRP ameliorated the clinical symptoms and hind paw swelling, with reduced arthritis scores compared with DEX- and PBS-treated CIA 


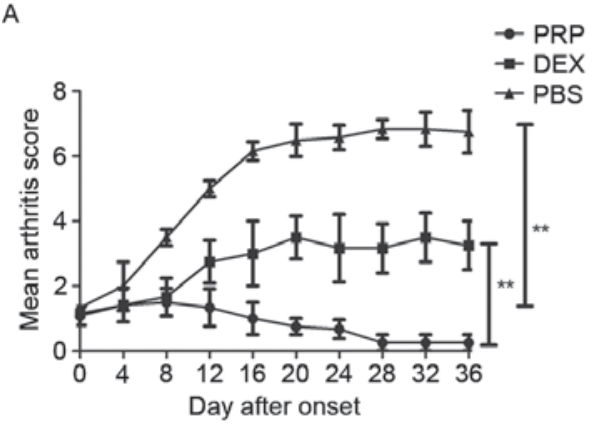

D

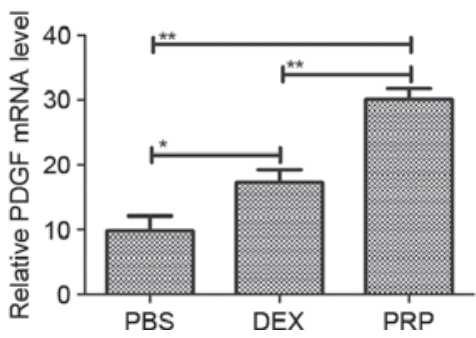

$B$

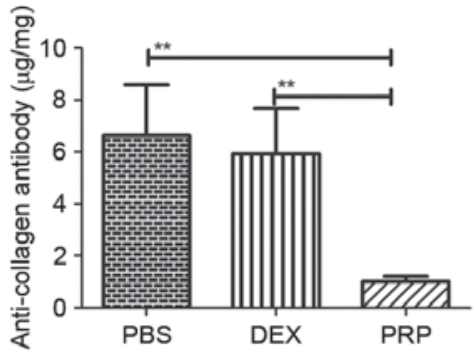

E

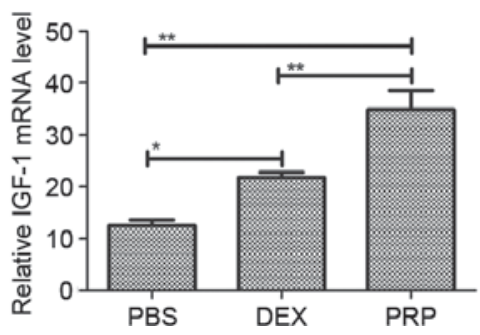





Figure 3. Assessment of the therapeutic effects of PRP in CIA mice. (A) Mice were given a subcutaneous injection of PRP (10 mg/kg), DEX or an equal volume of PBS. The arthritis scores were compared among non-treated $(n=9)$, DEX $(n=9)$ and PRP-treated $(n=9)$ groups during treatment. (B) Serum concentrations of anti-collagen immunoglobulin $\mathrm{G}$ in experimental mice were determined using ELISA. The expression of (C) VEGF, (D) PDGF, (E) IGF-1 and (F) TGF- $\beta$ was detected in synovial tissue in mice with CIA. Data are presented as the mean \pm the standard deviation of triplicate samples. ${ }^{*} \mathrm{P}<0.05$; ${ }^{* *} \mathrm{P}<0.01$. PRP, platelet-rich plasma; DEX, dexamethasone; PDGF, platelet-derived growth factor; IGF, insulin-derived growth factor; TGF, transforming growth factor; VEGF, vascular endothelial growth factor; CIA, collagen-induced arthritis.


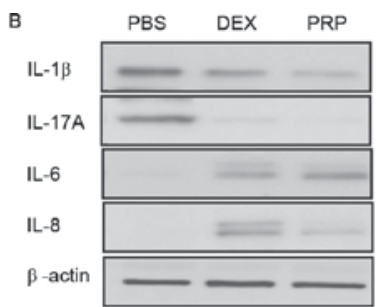

C

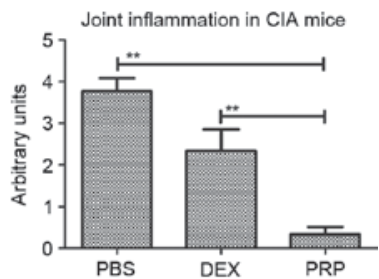

E

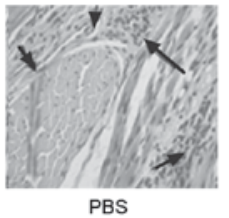

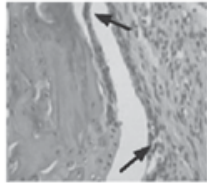

DEX

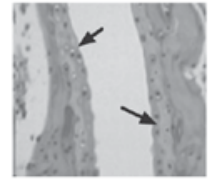

PRP

Figure 4. Analysis of RA-associated cytokines and histopathology of RA synovial grafts in CIA mice. (A) mRNA and (B) protein expression levels of IL-6, IL-8, MMP-3, IL-17, IL-1 $\beta$ and IL-17A in cartilage tissue were quantified using reverse transcription-quantitative polymerase chain reaction. The results are expressed as the $\mathrm{n}$-fold difference relative to $\beta$-actin. PRP treatment reduced (C) joint degeneration and (D) synovial proliferation in mice with CIA. (E) Healthy mouse and RA synovial tissue samples were stained with hematoxylin and eosin (magnification, $\mathrm{x} 200$ ). Data are presented as the mean \pm standard error of the mean. Tukey Kramer test; * ${ }^{* *}<0.01$. RA, rheumatoid arthritis; IL, interleukin; MMP, matrix metalloproteinase; PRP, platelet-rich plasma; CIA, collagen-induced arthritis; DEX, dexamethasone.

mice. In addition, the therapeutic effects of PRP were assessed by analyzing the humoral anti-collagen response. The serum concentration levels of anti-collagen type II IgG on day 72 were measured. The results in Fig. 3B demonstrated that a significant decrease in the serum level of anti-CII IgG was exhibited in PRP-treated RA mice compared with PBS- and DEX-treated groups ( $\mathrm{P}<0.01$ vs. control groups). Vascular endothelial growth factor (VEGF), platelet-derived growth factor (PDGF), insulin-like growth factor (IGF)-1 and transforming growth factor (TGF)- $\beta$ expression was assessed at the mRNA level in whole blood samples from RA mice, using RT-qPCR. The results of the present study indicated that VEGF, PDGF, IGF-1 and TGF- $\beta$ expression in peripheral whole blood was upregulated following treatment with PRP compared with PBS and DEX groups (Fig. 3C-F).

Analysis of the mechanism of PRP-associated benefits in RA mice. The therapeutic mechanism of PRP in RA synovial cells was analyzed in the present study. Target mRNA and protein expression levels were measured in synovial tissue from 
independent experiments following treatment with PRP. The results in Fig. 4A and B demonstrated that IL-6, IL-8, IL-17A, IL-1 $\beta$ mRNA expression was decreased in synovial tissue in PRP-treated mice compared with PBS-treated and DEX-treated groups. In addition, in order to observe alterations in RA synovial tissue following treatment with PRP, histological staining was used to analyze the therapeutic effects of PRP in mice with RA. As presented in Fig. 4C-E, the PRP-treated mice with RA exhibited marked reduction of synovial hyperplasia, inflammatory cell infiltration and destruction of cartilage and bone. In addition, it was observed that fewer infiltrating cells presented in the joints of the PRP-treated mice compared with PBS-treated and DEX-treated mice with CIA. The pathological symptoms of the PRP-treated CIA mice were attenuated. These results indicated that PRP may be an effective drug for RA therapy.

\section{Discussion}

The first description of the applications of PRP in biomedicine was reported in 1974 (25) and demonstrated differential alterations in the potency of platelet-aggregation inhibitors following pre-incubation in PRP. Further studies aimed to elucidate the biological mechanism underlying the effects of PRP, and potential applications as a therapeutic strategy for musculo-skeletal disorders, including ligament and tendon pathologies, and various cartilage pathologies, including RA and avascular necrosis $(26,27)$. PRP has been reported to be associated with numerous human diseases; in joint diseases, the application of autologous PRP has been observed to lead to decreased healing times in various clinical cases (28-30). In addition, a previous report suggested that the efficacy and feasibility of PRP treatment was notable in RA and avascular necrosis (31). However, although the application of PRP in the laboratory and in clinical practice has exhibited positive outcomes, the use of PRP has remained controversial, particularly its application as a treatment for RA and ankle cartilage pathology (32).

RA is an autoimmune inflammatory disorder which results in persistent synovitis with cartilage phlegmonosis and severe synovium destruction (33). Treatment with dexamethasone, golimumab, infliximab and adalimumab is frequently used in RA patients to reduce bone osteoporosis and manage synovium destruction. However, few reports have investigated the effects of treatment with PRP on RA. Lippross et al (34) demonstrated that inhibition of inflammatory mediators by PRP in RA led to symptomatic remission through modern molecular interventions and synovial repairmen (35). In addition, inhibition of inflammation and increasing advantageous cytokines by PRP has been evidenced to be a treatment for RA and osteoarthritis (26).

The present study investigated the therapeutic effects of PRP in CIA mice with RA. It was observed that treatment with PRP alleviated arthritis, reduced humoral and cellular immune responses, and led to beneficial effects on histological parameters as demonstrated by joint tissue histological staining. CIA RA mice treated with PRP exhibited downregulated expression of IL-6, IL-8, MMP-3, IL-17, IL-1 $\beta$, TNF- $\alpha$, RANKL and IFN- $\gamma$ in inflammatory tissue $(36,37)$. In addition, the serum concentration of anti-collagen was decreased in the
PRP-treated CIA mice. As the effect of PRP in the articular environment derives from an interaction with the pre-existing environment and other cells, and as certain surgical protocols involve the application of platelets and cells, several studies have also investigated the effect of PRP on mesenchymal stem cells of various origins (38-41). The stimulatory effects of treatment with PRP have been well-documented, originating from the action of promoting proliferation and chondrogenic differentiation, and thereby increasing the production of beneficial molecules for the maintenance of articular cartilage $(23,42)$. The results of the present study indicated that hypertrophy and leukocyte infiltration were suppressed following 4 weeks of treatment, which suggested that PRP treatment is beneficial for RA therapy.

Although a previous study has investigated the direct effects of different drugs on chondrocytes (43), further research is required to investigate the role of treatment with PRP in maintaining the cytokine homeostasis of the joint. The present study demonstrated that PRP markedly enhanced the activity of synovial cells and improved synovial angiogenesis. In conclusion, the results of the present study indicate that CIA mice treated with PRP exhibit beneficial effects on joint inflammation, cartilage destruction and bone damage.

\section{Acknowledgements}

The study was supported by the National Natural Science Foundation of China (grant no. 81501865).

\section{References}

1. Smyth NA, Haleem AM, Ross KA, Hannon CP, Murawski CD, Do HT and Kennedy JG: Platelet-rich plasma may improve osteochondral donor site healing in a rabbit model. Cartilage 7: 104-111, 2016.

2. Vannini F, Di Matteo B and Filardo G: Platelet-rich plasma to treat ankle cartilage pathology-from translational potential to clinical evidence: A systematic review. J Exp Orthop 2: 2, 2015.

3. Fu CJ, Sun JB, Bi ZG, Wang XM and Yang CL: Evaluation of platelet-rich plasma and fibrin matrix to assist in healing and repair of rotator cuff injuries: A systematic review and meta-analysis. Clin Rehabil 31: 158-172, 2017.

4. Wilson JJ, Lee KS, Chamberlain C, DeWall R, Baer GS, Greatens $M$ and Kamps N: Intratendinous injections of platelet-rich plasma: Feasibility and effect on tendon morphology and mechanics. J Exp Orthop 2: 5, 2015.

5. Zhang X, Wang J, Ren M, Li L, Wang Q and Hou X: A novel collagen/platelet-rich plasma (COL/PRP) scaffold: Preparation and growth factor release analysis. Cell Tissue Bank 17: 327-334, 2016.

6. Filardo G, Kon E, DI Matteo B, DI Marino A, Sessa A, Merli ML and Marcacci M: Leukocyte-poor PRP application for the treatment of knee osteoarthritis. Joints 1: 112-120, 2013.

7. Jang SJ, Kim JD and Cha SS: Platelet-rich plasma (PRP) injections as an effective treatment for early osteoarthritis. Eur J Orthop Surg Traumatol 23: 573-580, 2013.

8. Battaglia M, Guaraldi F, Vannini F, Buscio T, Buda R, Galletti S and Giannini S: Platelet-rich plasma (PRP) intra-articular ultrasound-guided injections as a possible treatment for hip osteoarthritis: A pilot study. Clin Exp Rheumatol 29: 754, 2011.

9. Hodge JA, Kawabata TT, Krishnaswami S, Clark JD, Telliez JB, Dowty ME, Menon S, Lamba M and Zwillich S: The mechanism of action of tofacitinib-an oral Janus kinase inhibitor for the treatment of rheumatoid arthritis. Clin Exp Rheumatol 34: 318-328, 2016.

10. van der Goes MC, Jacobs JW and Bijlsma JW: Rediscovering the therapeutic use of glucocorticoids in rheumatoid arthritis. Curr Opin Rheumatol 28: 289-296, 2016. 
11. van den Berg WB and Miossec P: IL-17 as a future therapeutic target for rheumatoid arthritis. Nat Rev Rheumatol 5: 549-553, 2009.

12. Haus E, Sackett-Lundeen L and Smolensky MH: Rheumatoid arthritis: Circadian rhythms in disease activity, signs and symptoms, and rationale for chronotherapy with corticosteroids and other medications. Bull NYU Hosp Jt Dis 70 (Suppl 1): S3-S10, 2012.

13. Souri M, Mokuda S, Inanami H, Osaki T, Takasugi $\mathrm{K}$ and Ichinose A: Non-autoimmune combined factor XIII A and B subunit deficiencies in rheumatoid arthritis patients treated with anti-interleukin-6 receptor monoclonal antibody (tocilizumab). Thromb Res 140: 100-105, 2016.

14. Chiu WC, Lai YP and Chou MY: Humanization and characterization of an anti-human TNF- $\alpha$ murine monoclonal antibody. PLoS One 6: e16373, 2011.

15. Weisman MH: TNF and anti-TNF treatment in rheumatoid arthritis (RA). What we know and what we still need to know. Ryumachi 37: 142-143, 1997.

16. Elliot MJ, Maini RN, Feldmann M, Long-Fox A, Charles $P$ Katasikis P, Brennan FM, Bijl H, Ghrayeb J and Woody JN: Treatment of rheumatoid arthritis with chimeric monoclonal antibodies to tumor necrosis factor alpha. Arthritis Rheum 58 (2 Suppl): S92-S101, 2008.

17. Segal B, Rhodus NL and Patel K: Tumor necrosis factor (TNF) inhibitor therapy for rheumatoid arthritis. Oral Surg Oral Med Oral Pathol Oral Radiol Endod 106: 778-787, 2008.

18. Gormeli G, Görmeli CA, Ataoglu B, Colak C, Aslantürk O and Ertem K: Multiple PRP injections are more effective than single injections and hyaluronic acid in knees with early osteoarthritis: A randomized, double-blind, placebo-controlled trial. Knee Surg Sports Traumatol Arthrosc 25: 958-965, 2017.

19. No authors listed: NIH requests information on care of laboratory animals. Am J Vet Res 67: 204-205, 2006.

20. Livak KJ and Schmittgen TD: Analysis of relative gene expression data using real-time quantitative PCR and the 2(-Delta Delta C(T)) method. Methods 25: 402-408, 2001

21. Nakajima H, Takamori H, Hiyama Y and Tsukada W: The effect of treatment with interferon-gamma on type II collagen-induced arthritis. Clin Exp Immunol 81: 441-445, 1990.

22. Stuart JM, Townes AS and Kang AH: Type II collagen-induced arthritis. Ann N Y Acad Sci 460: 355-362, 1985.

23. Bai F, Tian H, Niu Z, Liu M, Ren G, Yu Y, Sun T, Li S and Li D: Chimeric anti-IL-17 full-length monoclonal antibody is a novel potential candidate for the treatment of rheumatoid arthritis. Int J Mol Med 33: 711-721, 2014

24. Matsuno H, Yudoh K, Katayama R, Nakazawa F, Uzuki M Sawai T, Yonezawa T, Saeki Y, Panayi GS, Pitzalis C and Kimura T: The role of TNF-alpha in the pathogenesis of inflammation and joint destruction in rheumatoid arthritis (RA): A study using a human RA/SCID mouse chimera. Rheumatology (Oxford) 41: 329-337, 2002.

25. Gordon JL: Differential changes in potency of platelet-aggregation inhibitors after preincubation in platelet-rich plasma. Res Commun Chem Pathol Pharmacol 7: 221-224, 1974

26. Yilmaz S, Aksoy E, Doganci S, Yalcinkaya A, Diken AI and Cagli K: Autologous platelet-rich plasma in treatment of chronic venous leg ulcers: A prospective case series. Vascular 23: 580-585, 2015

27. Malahias MA, Chytas D, Babis GC and Nikolaou VS: Platelet-rich plasma guided injections: Clinical application in peripheral neuropathies. Front Surg 1: 41, 2014.

28. Apostol MI, Sawaya MR, Cascio D and Eisenberg D: Crystallographic studies of prion protein $(\mathrm{PrP})$ segments suggest how structural changes encoded by polymorphism at residue 129 modulate susceptibility to human prion disease. J Biol Chem 285 : 29671-29675, 2010.
29. Nisbet RM, Harrison CF, Lawson VA, Masters CL, Cappai R and Hill AF: Residues surrounding the glycosylphosphatidylinositol anchor attachment site of PrP modulate prion infection: Insight from the resistance of rabbits to prion disease. J Virol 84 6678-6686, 2010.

30. Silva JL, Gomes MP, Vieira TC and Cordeiro Y: PrP interactions with nucleic acids and glycosaminoglycans in function and disease. Front Biosci (Landmark Ed) 15: 132-150, 2010

31. San Sebastian KM, Lobato I, Hernández I, Burgos-Alonso N, Gomez-Fernandez MC, López JL, Rodríguez B, March AG, Grandes G and Andia I: Efficacy and safety of autologous platelet rich plasma for the treatment of vascular ulcers in primary care: Phase III study. BMC Fam Pract 15: 211, 2014.

32. Filardo G, Presti ML, Kon E and Marcacci M: Nonoperative biological treatment approach for partial achilles tendon lesion. Orthopedics 33: 120-123, 2010.

33. de Molon RS, Hsu C, Bezouglaia O, Dry SM, Pirih FQ, Soundia A, Cunha FQ, Cirelli JA, Aghaloo TL and Tetradis S: Rheumatoid Arthritis Exacerbates the Severity of Osteonecrosis of the Jaws (ONJ) in Mice. A Randomized, Prospective, Controlled Animal Study. J Bone Miner Res 31: 1596-1607, 2016.

34. Lippross S, Moeller B, Haas H, Tohidnezhad M, Steubesand N, Wruck CJ, Kurz B, Seekamp A, Pufe T and Varoga D: Intraarticular injection of platelet-rich plasma reduces inflammation in a pig model of rheumatoid arthritis of the knee joint. Arthritis Rheum 63: 3344-3353, 2011.

35. Tolusso B, Alivernini S, Gigante MR, Ferraccioli G and Gremese E: Biomolecular features of inflammation in obese rheumatoid arthritis patients: Management considerations. Expert Rev Clin Immunol 12: 751-762, 2016.

36. Catrina AI, Lampa J, Ernestam S, af Klint E, Bratt J, Klareskog L and Ulfgren AK: Anti-tumour necrosis factor (TNF)-alpha therapy (etanercept) down-regulates serum matrix metalloproteinase (MMP)-3 and MMP-1 in rheumatoid arthritis Rheumatology (Oxford) 41: 484-489, 2002.

37. Jeong JG, Kim JM, Cho H, Hahn W, Yu SS and Kim S: Effects of IL-1beta on gene expression in human rheumatoid synovial fibroblasts. Biochem Biophys Res Commun 324: 3-7, 2004.

38. Choy EH and Panayi GS: Cytokine pathways and joint inflammation in rheumatoid arthritis. N Engl J Med 344: 907-916, 2001

39. Stessuk T, Puzzi MB, Chaim EA, Alves PC, de Paula EV, Forte A, Izumizawa JM, Oliveira CC, Frei F and Ribeiro-Paes JT: Platelet-rich plasma (PRP) and adipose-derived mesenchymal stem cells: Stimulatory effects on proliferation and migration of fibroblasts and keratinocytes in vitro. Arch Dermatol Res 308: 511-520, 2016.

40. Tsuzuki N, Seo JP, Yamada K, Haneda S, Furuoka H, Tabata Y and Sasaki N: The effect of a gelatin $\alpha$-tricalcium phosphate sponge loaded with mesenchymal stem cells (MSC), bone morphogenic protein-2 and platelet-rich plasma (PRP) on equine articular cartilage defect. Can Vet J 54: 573-580, 2013.

41. Lin SS, Landesberg R, Chin HS, Lin J, Eisig SB and Lu HH: Controlled release of PRP-derived growth factors promotes osteogenic differentiation of human mesenchymal stem cells. Conf Proc IEEE Eng Med Biol Soc 1: 4358-4361, 2006.

42. Suzuki M, Tetsuka T, Yoshida S, Watanabe N, Kobayashi M, Matsui $\mathrm{N}$ and Okamoto T: The role of p38 mitogen-activated protein kinase in IL- 6 and IL-8 production from the TNF-alphaor IL-1beta-stimulated rheumatoid synovial fibroblasts. FEBS Lett 465: 23-27, 2000.

43. Drengk A, Zapf A, Sturmer E, Sturmer K and Frosch KH: Influence of platelet rich plasma (PRP) on chondrogenic differentiation and proliferation of chondrocytes (CC) and mesenchymal stem cells (MSC). J Stem Cells Regen Med 2: 180-181, 2007. 